\title{
Objective value on Apparent diffusion coefficient (ADC) map to categorize the intensity of diffusion-weighted imaging (DWI) restriction for prostate cancer detection on multiparametric prostate MRI
}

Thais Caldara Mussi ${ }^{1}$, Tatiana Martins ${ }^{1,2}$, Adriano Tachibana ${ }^{1}$, Pedro Nogueira Mousessian ${ }^{1}$, Ronaldo Hueb Baroni ${ }^{1}$

${ }^{1}$ Hospital Israelita Albert Einstein, São Paulo, SP, Brasil and ${ }^{2}$ Ecoar Medicina Diagnostica, Belo Horizonte, $M G$, Brasil

\section{ABSTRACT}

Purpose: To identify objective and subjective criteria on multiparametric prostate MRI that can be helpful for prostate cancer detection.

Materials and Methods: Retrospective study, IRB approved, including 122 patients who had suspicious lesion on MRI and who underwent prostate biopsy with ultrasonography (US)/MRI imaging fusion. There were 60 patients with positive biopsies and 62 with negative biopsies. MRI of these patients were randomized and evaluated independently by two blinded radiologists. The following variables were analyzed in each lesion: morphology, contours, T2 signal, diffusion restriction (subjective impression and objective values), hyper-enhancement, contact with transition zone or prostatic contour, prostatic contour retraction, Likert and PIRADS classification.

Results: Apparent diffusion coefficient (ADC) value was the best predictor of positivity for prostate cancer, with mean value of 1.08 (SD 0.20) and $1.09 \mathrm{~mm} 2 / \mathrm{sec}$ (SD 0.24) on negative biopsies and 0.81 (SD 0.22) and $0.84 \mathrm{~mm} 2 / \mathrm{sec}$ (SD 0.22) on positive biopsies for readers 1 and 2, respectively ( $p<0.001$ in both analysis). For the others categorical variables evaluated the best AUC for reader 1 was subjective intensity of diffusion restriction (AUC of 0.74) and for reader 2 was hyper-enhancement (AUC of 0.65), all inferior comparing to the value of $\mathrm{ADC}$ map. Interobserver agreement ranged from 0.13 to 0.75 , poor in most measurements, and good or excellent (kappa $>0.6$ ) only in lesion size and ADC values.

Conclusions: Diffusion restriction with lower ADC-values is the best parameter to predict cancer on MRI prior to biopsy. Efforts to establish an ADC cutoff value would improve cancer detection, especially for less experience reader.

\section{ARTICLE INFO}

\section{Keywords:}

Magnetic Resonance Imaging;

Prostatic Neoplasms; Prostate

Int Braz J Urol. 2018; 44: 882-91

Submitted for publication:

January 23, 2018

Accepted after revision:

May 13, 2018

Published as Ahead of Print: June 18, 2018

\section{INTRODUCTION}

Magnetic resonance imaging (MRI) of the prostate is an important tool to detect cancer (1$3)$, stage the disease (4), estimate cancer aggressiveness $(5,6)$ and follow-up men with previous negative biopsies or on active surveillance $(7,8)$.
However, prostate MRI is a challenging exam, with limitations, so its description is usually performed in probability grades of having a clinically significant (CS) cancer. A Likert scale is a subjective scale firstly described by a neuroscientist and is used to grade something in levels of certainty (9). In prostate MRI the Likert scale is 
used to describe the probability of having prostate cancer, usually in a 5-point scale $(10,11)$. More recently, in an attempt to standardize the methodology and make it more objective, a multidisciplinary group published the PI-RADS (Prostate Imaging Reporting and Data System) in 2012 (12), and an updated version in 2015 (13). PI-RADS version 2 also classifies prostate lesions in a 5-point scale of probability of having a significant cancer. PI-RADS is based on subjective features of prostatic lesions, and the version 2 uses the diffusionweighted image sequence as the dominant to categorize a lesion in the peripheral zone, and the T2-weighted imaging morphology (including heterogeneity) as the dominant sequence for lesion in transition zone. If a lesion is indeterminate, positive enhancement is used to differentiate from high probability of CS cancer in the peripheral zone and the grade of diffusion restriction is used similarly on the transition zone (12).

Nowadays, PI-RADS version 2 is widely used to standardize the communication between radiologists and urologists and also to make MRI evaluation and reports more uniform and reproducible among radiologists. It is able to improve detection, localization, characterization, and risk stratification (13). Nevertheless, some studies show low to moderate rates of interobserver agreement, even for experienced reader $(14,15)$.

The purpose of this study is to identify objective and subjective criteria on multiparametric prostate MRI that can help in the detection of prostate cancer and, therefore, improve MRI results.

\section{MATERIALS AND METHODS}

\section{Study design}

Retrospective study, institutional review board approved. We searched in our database for patients who had suspicious lesions on MRI, with prostate MRI report of high or very high probability for CS prostate cancer (PI-RADS 4 and 5) and who also underwent prostate biopsy with ultrasonography (US) / MRI imaging fusion in our institution.

Between March, 2015 and January, 2016 we found 60 patients who had PI-RADS 4 or 5 findings on MRI, including peripheral zone and transition zone lesions, with positive biopsy results for prostate cancer. Then, to eliminate a selection bias, we also included 62 patients with suspicious lesions on MRI (PI-RADS 4 and 5), but with negative biopsy results. The maximal interval between MRI and biopsies was 6 months for all patients.

If a patient had more than one suspicion lesion, the highest PI-RADS scoring lesion was counted for each patient. Images of these 122 patients were anonymized, randomized and evaluated independently by two radiologists, blinded to the clinical and histopathological results (reader 1, a board-certified radiologist with 10 years of experience in general radiology and 5 years of experience in prostate MRI, and reader 2, a boardcertified radiologist with 15 years of experience in general radiology and 2 years of experience in prostate MRI). To be sure that both radiologists were reading the same lesion, a third radiologist reviewed the MRI and biopsy reports, and gave them the lesion localization (prostate lobe, prostate zone and image number on T2-weighted (T2W) imaging, matched to diffusion-weighted imaging [DWI] and dynamic contrast enhanced sequences [DCE]). The slide presentation with the imaging localization was used just to show the lesion that should be considered, however the entire exam was evaluated in a PACS (Picture Archiving and Communication System) station (KODAK / Carestream; Carestream Health, Rochester, New York, USA) independently by both radiologists. The following variables were analyzed for each lesion: morphology (wedge-shape, oval or round), contours (circumscribed, partially defined or ill-defined), T2W signal intensity (hypointense or marked hypointense), T2W signal homogeneity (homogeneous or heterogeneous), subjective diffusion restriction (mild, moderate or marked), objective value measured on apparent diffusion coefficient (ADC) map, hyper-enhancement (absent, mild, moderate or marked), contact with transition zone (absent or present) or prostatic contour (absent or present), and prostatic contour retraction (absent or present). Each lesion was also classified based on Likert and PI-RADS version 2 scales. 


\section{MRI protocol}

All patients underwent MRI on a 3-Tesla scanner: Magnetom Prisma (Siemens Medical Solutions, Erlangen, Germany) or Discovery MR 750W (GE Healthcare, Little Chalfont, United Kingdom with a phased array coil and without an endorectal coil. A routine protocol including triplanar $\mathrm{T} 2 \mathrm{~W}$ imaging using the parameters: repetition time (msec) / echo time (msec), 4700-5200 / 140-160; section thickness, $3 \mathrm{~mm}$; field of view, 180 x 180; matrix 256 × 256; acceleration factor of two; and six averages. DWI were acquired with b-values of 50, 400, 800 and $1500 \mathrm{sec} / \mathrm{mm} 2$; and the ADC map was constructed based in a mono-exponential approach. DCE imaging were performed using the parameters: repetition time (msec) / echo time (msec), 3.4-3.5 / 1.4; section thickness, $3 \mathrm{~mm}$; field of view, 250; matrix $224 \times 224$; acquiring 14 sequences with 10 seconds of temporal resolution. Extracellular gadolinium-based contrast media (Magnevist, Bayer, Leverkusen, Germany) was injected at a dose of $0.2 \mathrm{cc} / \mathrm{Kg}$ and a rate of 2 cc / sec.

\section{Biopsy protocol}

As reference standard, the transrectal prostate biopsy with MRI / US fusion and additional samples of suspicious areas was adopted. US-guided biopsies were performed using either an Aplio 500 with Smart Fusion (Toshiba Medical System Corporation, Minato, Tokyo, Japan) or a LOGIC E9 with imaging fusion software (GE Healthcare, Little Chalfont, United Kingdom). One out of ten radiologists with at least 4 years of experience in prostate biopsy with imaging fusion MRI / US performed the biopsy. A cancer was defined as CS if Gleason score $\geq 3+4$.

\section{Imaging evaluation}

Radiologists evaluated the images using a workstation (Carestream, Rochester, New York). All sequences of the exam were interpreted in a single session and the selected parameters of that study were evaluated. ADC values were measured on the ADC map with a round $\mathrm{ROI}$ in the lesion in the transverse plane.

\section{Statistical analysis}

We performed a histogram analysis and Shapiro-Wilk test to verify the distribution. Numeric variables with normal distribution were described as mean and standard deviation (SD), and numeric variables with no normal distribution were described as median and interquartile intervals (IQR). Categorical variables were described by absolute and relative frequencies.

The interobserver agreement was calculated using Cohen's Kappa statistics (linear weights for categorical variables and quadratic weights for ordinal variable). It was defined as: excellent $(\mathrm{k} \geq 0.81)$, good $(\mathrm{k}=0.61-0.80)$, moderate $(\mathrm{k}=0.41-0.60)$, fair $(\mathrm{k}=0.21-0.40)$ and poor $(\mathrm{k} \leq 20)$.

To study the association between the explicative measurements and the biopsy results we used binominal logistic models and the discrimination was verified with area under the curve (AUC) receiver operating characteristic (ROC). Multiple model was acquired using stepwise process in both direction starting with a null model to a saturated model.

Analyses were performed using the software R 3.1.3 ( $\mathrm{R}$ Core Team, 2015). The level for statistical significance was set at 5\%.

\section{RESULTS}

A total of 122 patients were enrolled in our study. Of the 60 positive biopsy results, 9 (15\%) had Gleason score $3+3$ and 51 (85\%) had Gleason score $\geq 3+4$. Median time between MRI and biopsy was 21 days. A median of 3 additional samples was obtained in each suspicious lesion on MRI.

Among all variables independently analyzed, ADC value was the best predictor of positivity for CS-prostate cancer on biopsy, with mean value of $1.08 \mathrm{~mm}^{2} / \mathrm{sec}$ (SD 0.20) on negative biopsies and $0.81 \mathrm{~mm} 2 / \mathrm{sec}$ (SD 0.22) on positive biopsies for reader 1 , and $1.09 \mathrm{~mm}^{2} / \mathrm{sec}$ (SD 0.24) on negative biopsies and $0.84 \mathrm{~mm}^{2} / \mathrm{sec}$ (SD 0.22) on positive biopsies for reader 2 ( $p<0.001$ in both analysis). AUC was 0.82 and 0.80 for reader 1 and 2 , respectively (Table-1).

For categorical variables (morphology, contours, signal in $\mathrm{T} 2 \mathrm{~W}$, subjective intensity of 
diffusion restriction, hyper-enhancement, contact with surgical and prostatic capsule, and retraction of prostatic contour), the best AUC for reader 1 (Table-2) was subjective intensity of diffusion restriction (AUC of 0.74) and for reader 2 (Table-3) was hyper-enhancement (AUC of 0.65), all inferior comparing to the value of ADC map. Table-4 shows sensitivities, specificities, accuracies, positive and negative predictive for tumor, in presence of objective measurement of ADC value, being the most important variable that correlates with biopsy (Table-5) (Figure-1).

Boxplots graphics shows that subjective impression to categorize the intensity of restriction (mild, moderate and marked) had direct strong negative correlation with ADC values, with coefficients of -0.83 (confidence interval of 95\%: -0.90 to -0.74 ) for reader 1 , and -0.64

Table 1 - Numeric variables included in the study.

\begin{tabular}{cccccc}
\hline & VARIABLES & NEGATIVE & POSITIVE & AUC $(95 \% \mathrm{CI})$ & P VALUE \\
\hline \multirow{2}{*}{ Reader 1} & Size $(\mathrm{mm})$ & $7.00[5.00 ; 11.00]$ & $9.50[7.75 ; 12.00]$ & $0.64(0.54-0.74)$ & 0.048 \\
& ADC-Value & $1.08(0.20)$ & $0.81(0.22)$ & $0.82(0.73-0.91)$ & $<0.001$ \\
& Likert score & $3.00[2.00 ; 3.00]$ & $4.00[3.00 ; 5.00]$ & $0.78(0.70-0.85)$ & $<0.001$ \\
& PIRADS score & $3.00[2.00 ; 3.00]$ & $4.00[3.00 ; 4.00]$ & $0.77(0.70-0.85)$ & $<0.001$ \\
\multirow{2}{*}{ Reader 2 } & Size $(\mathrm{mm})$ & $10.00[6.00 ; 14.00]$ & $14.00[9.75 ; 16.00]$ & $0.65(0.55-0.75)$ & 0.010 \\
& ADC-Value & $1.09(0.24)$ & $0.84(0.22)$ & $0.80(0.70-0.89)$ & $<0.001$ \\
& Likert score & $4.00[4.00 ; 5.00]$ & $5.00[4.00 ; 5.00]$ & $0.59(0.50-0.68)$ & 0.054 \\
& PIRADS score & $4.00[4.00 ; 4.00]$ & $4.00[4.00 ; 5.00]$ & $0.62(0.54-0.71)$ & 0.010 \\
\hline
\end{tabular}

AUC = Area under the ROC Curve; $\mathbf{9 5} \% \mathbf{C l}=95 \%$ confidence intervals; $\mathbf{O R}=$ estimated odds ratio

values of both readers in the features that had clinically significant differences on independent analysis.

Interobserver agreement ranged from 0.13 to 0.75 , poor in most measurements, and good or excellent (kappa > 0.6) only in lesion size and ADC values (both numeric variables).

\section{Multiple models}

Multiple models analyses were obtained independently for readers 1 and 2 . For reader 1 , variables that remained significantly associated with tumor on biopsy, in presence of ADC value, were: contact with prostatic contour, subjective restriction diffusion on ADC map (moderate and marked), and lesion contours partially defined or ill defined. For reader 2, none of the variables were significant associated with positive biopsy (confidence interval of 95\%: -0.75 to -0.51 for reader 2). However, this association was stronger for reader 1 than for reader 2, who had high overlap on $\mathrm{ADC}$ values measurements for subjective categorization of moderate and marked diffusion restriction (Figure-2).

\section{DISCUSSION}

Our study showed that objective value of lesion's diffusion restriction, measured on ADC map, is the best predictive variable for prostate cancer. For the more experienced reader, the subjective value of ADC (moderate and marked) also had significant correlation with positive biopsies. However, for less experienced reader, the subjective variable had high overlap and showed no correlation with positive biopsies on multivariate analysis. 
Figure 1 - A nodule in the posterior right midgland of the peripheral zone $(A)$. Reader 1 described as mild restriction diffusion on ADC map and diffusion (B and C) and no hyperenhancement (D), and final Likert and PIRADS score 3. Reader 2 described as moderate diffusion and hyperenhancement, and final Likert and PIRADS scores 5. Biopsy was negative for neoplasia.

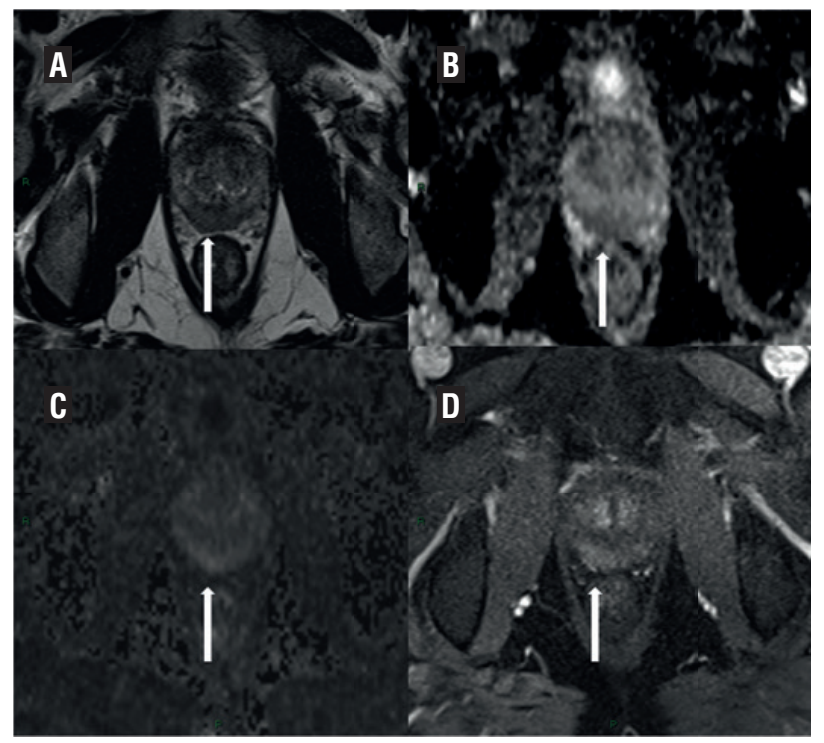

Prostate MRI is a method routinely performed to detect prostate cancer prior to first biopsy or after a negative biopsy $(1,2,7)$. A structured prostate report is a major contribution for prostate MRI examination, and represents the initial steps to do a widely available method to detect prostate cancer. However, it is a challenging method with low to moderate rates of interobserver agreement even with standardized methodology $(14,15)$.

It is already known that there is a correlation between the grade of diffusion restriction in the prostate and the presence of cancer (1620). Our study shows that the subjective graduation of restricted diffusion can be misclassified for less experience readers, what could imply the final categorization of a prostatic lesion on MRI.

The variability in the subjective analysis probably explains the low rates of interobserver agreement. Median ADC values in category moderate and marked was $0.89 \mathrm{~mm} 2 / \mathrm{sec}$ (IQR 0.88 - 0.96) and $0.62 \mathrm{~mm} 2 / \mathrm{sec}$ (IQR 0.54 - 0.72), respectively, for reader 1 , and $1.02 \mathrm{~mm} 2 / \mathrm{sec}$ (IQR 0.86 - 1.15) and $0.76 \mathrm{~mm} 2 / \mathrm{sec}$ (IQR 0.63 - 0.96),

Figure 2 - Graphics presented in boxplot showing the correlation of subjective ADC map grade and the objective ADC map value.

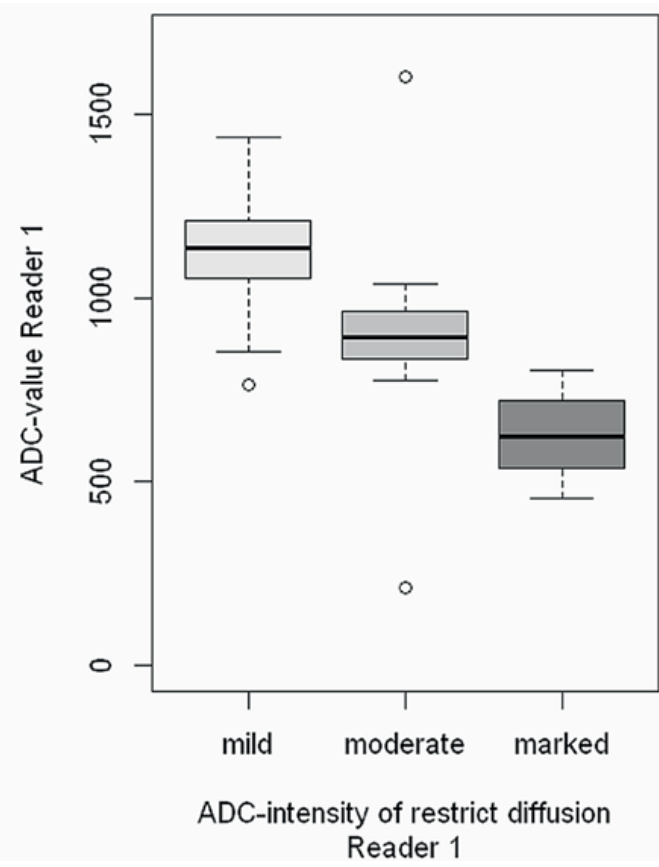

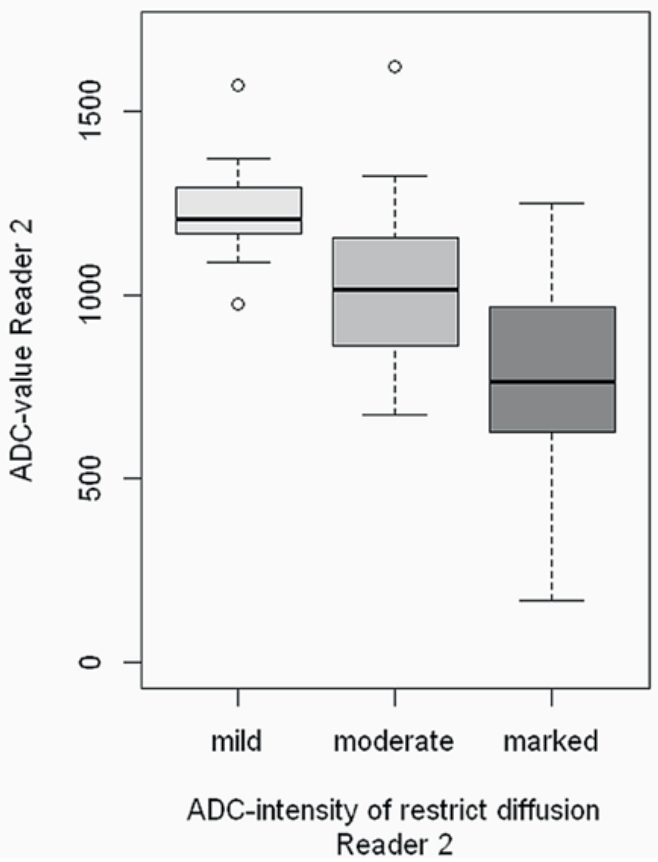


Table 2 - Categoric variable for reader 1 in absolute number (\%).

\begin{tabular}{lccccc}
\hline VARIABLES & CLASS & NEGATIVE $(\mathrm{n}=62)$ & POSITIVE $(\mathrm{n}=60)$ & AUC (95\%Cl) & P VALUE \\
\hline Morphology & 1-linear / V-shaped & $11(17.7)$ & $3(5.0)$ & $0.62(0.54-0.71)$ & \\
& 2-oval & $13(21.0)$ & $23(38.3)$ & & 0.011 \\
3-nodular & $38(61.3)$ & $34(56.7)$ & & 0.086 \\
Contours & 1-well defined & $28(45.2)$ & $17(28.3)$ & $0.59(0.50-0.68)$ & \\
2-partially defined & $26(41.9)$ & $32(53.3)$ & & 0.081 \\
Signal in T2 & 3-undefined & $8(12.9)$ & $11(18.3)$ & & 0.142 \\
1-hypo & $43(69.4)$ & $26(43.3)$ & $0.63(0.54-0.72)$ & \\
Signal in T2 & 2-marked hypo & $19(30.6)$ & $34(56.7)$ & & 0.004 \\
& 1-homogeneous & $18(29.0)$ & $36(60.0)$ & $0.65(0.57-0.74)$ & \\
ADC- intensity of & 2-heterogeneous & $44(71.0)$ & $24(40.0)$ & & 0.001 \\
diffusion restriction & 1-mild & $39(62.9)$ & $12(20.0)$ & $0.74(0.66-0.83)$ & \\
2-moderate & $17(27.4)$ & $26(43.3)$ & & $<0.001$ \\
Hyper-enhancement & 3-marked & $6(9.7)$ & $22(36.7)$ & & $<0.001$ \\
& 0-absent & $8(15.1)$ & $7(12.7)$ & $0.55(0.44-0.65)$ & \\
& 1-mild & $19(35.8)$ & $17(30.9)$ & & 0.971 \\
2-moderate & $15(28.3)$ & $15(27.3)$ & & 0.833 \\
Contact with surgical & 3-marked & $11(20.8)$ & $16(29.1)$ & & 0.434 \\
capsule & 0-no & $21(33.9)$ & $18(30.0)$ & $0.52(0.44-0.60)$ & \\
Contact with prostatic & 1-yes & $41(66.1)$ & $42(70.0)$ & & 0.647 \\
contours & 0-no & $23(37.1)$ & $10(16.7)$ & $0.60(0.53-0.68)$ & \\
Retraction of prostatic & 1-yes & $39(62.9)$ & $50(83.3)$ & & 0.013 \\
contours & 0-no & $58(96.7)$ & $52(94.5)$ & $0.51(0.47-0.55)$ & \\
\hline & 1-yes & $2(3.3)$ & $3(5.5)$ & & 0.581 \\
\hline
\end{tabular}

AUC = Area under the ROC Curve; $\mathbf{9 5} \% \mathbf{C I}=95 \%$ confidence intervals; $\mathbf{O R}=$ estimated odds ratio

respectively, for reader 2 . Note that for reader 2 there is an interpolation for moderate and marked restriction diffusion ranging from value from 0.86 $\mathrm{mm}^{2} / \mathrm{sec}$ to $0.96 \mathrm{~mm}^{2} / \mathrm{sec}$ (Figure-2). We believe that an objective cutoff for ADC map value, included in routine practice, would increase interobserver agreement to categorize lesions with mild, moderate and marked restriction diffusion, contributing to the standardize methodology become more reproducible. To the best of our knowledge, there is no data suggesting using objective ADC map values to categorize the grade of restriction diffusion.

There are some factors described that can affect the ADC measurements, such as b values, respiration conditions, field strength, vendor and other technical parameters (21). On the other hand, Sadinski et al. demonstrated, comparing ADC maps between two consecutive scans of same patient, that the reproducibility of ADC measurements in prostate is reasonable, suggesting that quantitative values obtained in DWI-MRI of prostate cancer are reproducible (22). Our study found similar values on ADC map for positive and negative prostate cancer biopsies $(0.81 \mathrm{~mm} 2 / \mathrm{sec}$ and $1.08 \mathrm{~mm} 2 / \mathrm{sec}$ for reader 1 , and $0.84 \mathrm{~mm} 2$ / sec and $1.09 \mathrm{~mm} 2$ / sec for reader 2, respectively), compared to literature, that ranges from $0.74 \mathrm{~mm} 2$ / sec (SD 0.15) to $0.80 \mathrm{~mm}^{2} / \mathrm{sec}$ (SD 0.25) for 
Table 3 - Categoric variable for reader 2 in absolute number (\%).

\begin{tabular}{|c|c|c|c|c|c|}
\hline VARIABLES & CLASS & NEGATIVE (n=62) & POSITIVE (n=60) & AUC $(95 \% \mathrm{Cl})$ & P VALUE \\
\hline \multirow[t]{3}{*}{ Morphology } & 1-linear / v-shaped & $10(16.1)$ & $9(15.0)$ & $0.54(0.45-0.63)$ & \\
\hline & 2-oval & $19(30.6)$ & $23(38.3)$ & & 0.593 \\
\hline & 3-nodular & $33(53.2)$ & $28(46.7)$ & & 0.911 \\
\hline \multirow[t]{3}{*}{ Contours } & 1-well defined & $30(48.4)$ & $16(26.7)$ & $0.62(0.52-0.71)$ & \\
\hline & 2-partially defined & $25(40.3)$ & $36(60.0)$ & & 0.014 \\
\hline & 3-undefined & $7(11.3)$ & $8(13.3)$ & & 0.206 \\
\hline \multirow[t]{2}{*}{ Signal in T2 } & 1-hypo & $43(69.4)$ & $35(58.3)$ & $0.56(0.47-0.64)$ & \\
\hline & 2-marked hypo & $19(30.6)$ & $25(41.7)$ & & 0.206 \\
\hline \multirow[t]{3}{*}{ Signal in T2 } & 1-homogeneous & $42(67.7)$ & $39(65.0)$ & $0.51(0.43-0.60)$ & \\
\hline & 2-heterogeneous & $20(32.3)$ & $21(35.0)$ & & 0.749 \\
\hline & 1-mild & $18(29.0)$ & $6(10.0)$ & $0.64(0.55-0.73)$ & \\
\hline \multirow{2}{*}{$\begin{array}{l}\text { ADC- intensity of } \\
\text { diffusion restriction }\end{array}$} & 2-moderate & $23(37.1)$ & $20(33.3)$ & & 0.088 \\
\hline & 3-marked & $21(33.9)$ & $34(56.7)$ & & 0.004 \\
\hline \multirow[t]{4}{*}{ Hyper-enhancement } & 0 -absent & $9(17.0)$ & $3(5.4)$ & $0.65(0.55-0.75)$ & \\
\hline & 1-mild & $7(13.2)$ & $10(17.9)$ & & 0.079 \\
\hline & 2-moderate & $12(22.6)$ & $24(42.9)$ & & 0.018 \\
\hline & 3-marked & $25(47.2)$ & $19(33.9)$ & & 0.261 \\
\hline \multirow{2}{*}{$\begin{array}{l}\text { Contact with surgical } \\
\text { capsule }\end{array}$} & 0-no & $20(32.3)$ & $20(33.3)$ & $0.51(0.42-0.59)$ & \\
\hline & 1 -yes & $42(67.7)$ & $40(66.7)$ & & 0.899 \\
\hline \multirow{2}{*}{$\begin{array}{l}\text { Contact with prostatic } \\
\text { contours }\end{array}$} & $0-$ no & $21(33.9)$ & $7(11.7)$ & $0.61(0.54-0.68)$ & \\
\hline & 1-yes & $41(66.1)$ & $53(88.3)$ & & 0.005 \\
\hline \multirow{2}{*}{$\begin{array}{l}\text { Retraction of prostatic } \\
\text { contours }\end{array}$} & 0 -no & $51(92.7)$ & $52(94.5)$ & $0.51(0.46-0.56)$ & \\
\hline & 1 -yes & $4(7.3)$ & $3(5.5)$ & & 0.697 \\
\hline
\end{tabular}

AUC = Area under the ROC Curve; $\mathbf{9 5} \% \mathbf{C l}=95 \%$ confidence intervals; $\mathbf{O R}=$ estimated odds ratio

positive prostate cancer biopsies and $1.35 \mathrm{~mm}^{2} /$ sec (SD 0.31) to $1.48 \mathrm{~mm} 2 /$ sec (SD 0.29) for negative biopsies $(18,19)$, corroborating that ADC map value can be reproducible. Also, it has been already shown that using a parameter to normalize the ADC measurement (as the normal parenchyma or muscle), the $\mathrm{ADC}$ value can be reproducible among different scanners (21).

All others variables included in this study (size, Likert and PI-RADS classifications, morphology, contours, signal in T2W, contact with surgical capsule and prostatic contours, prostatic contours retraction and hyper-enhancement) were less or no important comparing to the intensity of diffusion restriction on $\mathrm{ADC}$ map.
Rosenkrantz et al. (23) recently proposed adjustments in PI-RADS version 2: in transition zone upgrade score 3 to 4 based on diffusion restriction score of 4 or modified dynamic contrast enhanced positive when incorporating new criteria, and in transition or peripheral zones upgrade score 4 to 5 based on size of 10-14 mm. Our study also showed that intensity of diffusion restriction is the most important variable that correlates with cancer, even in transition zone, and indeed our present observation provides a solid background to upgrade the PI-RADS score 3 to 4 when there is moderate diffusion restriction instead of considering only marked diffusion restriction. Also, size of the lesions had a median value of $9.5 \mathrm{~mm}$ and 
Table 4 - Diagnostic measurements.

\begin{tabular}{|c|c|c|c|c|c|c|}
\hline VARIABLE & POSITIVE & SENSITIVITY & SPECIFICITY & ACCURACY & PPV & NPV \\
\hline \multicolumn{7}{|l|}{ Reader 1} \\
\hline Size (mm) & $\geq 7.5$ & 0.75 & 0.53 & 0.64 & 0.61 & 0.69 \\
\hline Morphology & oval or nodular & 0.95 & 0.18 & 0.56 & 0.53 & 0.79 \\
\hline Contour & $\begin{array}{l}\text { Partially ill-defined or ill- } \\
\text { defined }\end{array}$ & 0.72 & 0.45 & 0.58 & 0.56 & 0.62 \\
\hline Signal in T2 & marked hypo & 0.57 & 0.69 & 0.63 & 0.64 & 0.62 \\
\hline Signal in T2 & heterogeneous & 0.40 & 0.29 & 0.34 & 0.35 & 0.33 \\
\hline ADC - subjective & moderate or marked & 0.80 & 0.63 & 0.71 & 0.68 & 0.76 \\
\hline$A D C$ - value & $\geq 1010.5$ & 0.16 & 0.29 & 0.23 & 0.17 & 0.27 \\
\hline Early enhancement & marked & 0.29 & 0.79 & 0.54 & 0.59 & 0.52 \\
\hline Contact with prostatic contour & yes & 0.83 & 0.37 & 0.60 & 0.56 & 0.70 \\
\hline Likert & 4 or 5 & 0.55 & 0.85 & 0.70 & 0.79 & 0.66 \\
\hline PI-RADS & 4 or 5 & 0.55 & 0.85 & 0.70 & 0.79 & 0.66 \\
\hline \multicolumn{7}{|l|}{ Reader 2} \\
\hline Size (mm) & $\geq 7.5$ & 0.90 & 0.35 & 0.62 & 0.57 & 0.79 \\
\hline Morphology & Nodular & 0.47 & 0.47 & 0.47 & 0.46 & 0.48 \\
\hline Contour & $\begin{array}{l}\text { Partially ill-defined or ill- } \\
\text { defined }\end{array}$ & 0.73 & 0.48 & 0.61 & 0.58 & 0.65 \\
\hline Signal in T2 & marked hypo & 0.42 & 0.69 & 0.56 & 0.57 & 0.55 \\
\hline Signal in T2 & heterogeneous & 0.35 & 0.68 & 0.52 & 0.51 & 0.52 \\
\hline ADC - subjective & marked & 0.57 & 0.66 & 0.61 & 0.62 & 0.61 \\
\hline$A D C$ - value & $\geq 910$ & 0.30 & 0.12 & 0.20 & 0.22 & 0.17 \\
\hline Early enhancement & mild, moderate or marked & 0.95 & 0.17 & 0.57 & 0.55 & 0.75 \\
\hline Contact with prostatic contour & yes & 0.88 & 0.34 & 0.61 & 0.56 & 0.75 \\
\hline Likert & 5 & 0.60 & 0.58 & 0.59 & 0.58 & 0.60 \\
\hline PI-RADS & 5 & 0.35 & 0.89 & 0.62 & 0.75 & 0.59 \\
\hline
\end{tabular}

$\mathbf{P P V}=$ positive predictive value; $\mathbf{N P V}=$ negative predictive value

Table 5 - Multiple models to identify positive biopsy.

\begin{tabular}{lcccc}
\hline & COEFFICIENTS & ESTIMATE & OR $(95 \% \mathrm{Cl})$ & P VALUE \\
\hline Reader 1 & Intercept & -4.09 & & $<0.001$ \\
AUC & Contact with prostatic contour (present) & 1.42 & $4.14(1.50-11.43)$ & 0.006 \\
$0.82(0.74-0.89)$ & ADC - Intensity moderate or marked & 2.19 & $8.93(3.43-23.29)$ & $<0.001$ \\
& Contours (partially defined or ill-defined) & 1.09 & $2.98(1.17-7.58)$ & 0.022 \\
Reader 2 & Morphology (oval or round) & 1.14 & $3.13(0.65-14.96)$ & 0.153 \\
AUC & Intercept & 4.77 & & 0.001 \\
$0.82(0.74-0.91)$ & ADC - value & -0.01 & $0.995(0.993-0.997)$ & $<0.001$ \\
& Contact with surgical capsule (present) & -0.95 & $0.38(0.13-1.13)$ & 0.083 \\
& Contours (partially defined or ill-defined) & 0.89 & $2.42(0.88-6.65)$ & 0.086 \\
\hline
\end{tabular}


$14 \mathrm{~mm}$ in positive biopsies for readers 1 and 2, respectively, and did not correlated with positive biopsy in multiple models analyses. So, we agree that a $10 \mathrm{~mm}$ threshold would be a better cutoff to differentiate scores 4 and 5.

Our study has some limitations: first, we had only two readers with different levels of experience. Second, the readers evaluated only the previously specified lesions and not the whole gland; we chose this methodology because the reference standard used was the fusion biopsy (using US and RM images) and we wanted to be sure that the analyzed lesion was the biopsied one. Also, we aimed to compare the interobserver agreement and assess the variables for the same abnormality. Third, we did not analyze lesions in the peripheral and transition zones in subgroups. Finally, we did not measure the relative ADC map value.

\section{CONCLUSIONS}

Diffusion restriction with lower ADCvalues is the best parameter to predict cancer on multiparametric MRI prior to biopsy. Efforts to establish an ADC cutoff value would improve cancer detection, especially for less experience readers. Also, developing ADC as a quantitative imaging marker would allow better detection of prostate cancer by reducing inter-radiologist subjectivity, improving MRI results and therefore avoiding unnecessary biopsy, reducing overdiagnosis and overtreatment of prostate cancer.

\section{Clinical Relevance}

Prostate MRI is evolving for the diagnosis of cancer. There is still debate in literature to prove the best method for diagnosis, but none of them include ADC objective values to make the differentiation between benign and malignant lesions.

\section{ACKNOWLEDGEMENT}

We thank Ana Carolina Cintra Nunes Mafra for helping with the statistical analyses.

\section{ETHICAL APPROVAL}

IRB approved.

\section{CONFLICT OF INTEREST}

None declared.

\section{REFERENCES}

1. Ahmed HU, El-Shater Bosaily A, Brown LC, Gabe R, Kaplan R, et al. Diagnostic accuracy of multi-parametric MRI and TRUS biopsy in prostate cancer (PROMIS): a paired validating confirmatory study. Lancet. 2017;389(10071):815-22.

2. Nam RK, Wallis CJ, Stojcic-Bendavid J, Milot L, Sherman C, Sugar L, et al. A Pilot Study to Evaluate the Role of Magnetic Resonance Imaging for Prostate Cancer Screening in the General Population. J Urol. 2016;196:361-6.

3. Mussi TC, Garcia RG, Queiroz MR, Lemos GC, Baroni RH Prostate cancer detection using multiparametric 3 - tesla MRI and fusion biopsy: preliminary results. Int Braz J Urol. 2016;42:897-905.

4. Pasoglou V, Larbi A, Collette L, Annet L, Jamar F, Machiels JP, et al. One-step TNM staging of high-risk prostate cancer using magnetic resonance imaging (MRI): toward an upfront simplified "all-in-one" imaging approach? Prostate. 2014;74:469-77.

5. Moore CM, Ridout A, Emberton M. The role of MRI in active surveillance of prostate cancer. Curr Opin Urol. 2013;23:261-7.

6. Moore CM, Petrides N, Emberton M. Can MRI replace serial biopsies in men on active surveillance for prostate cancer? Curr Opin Urol. 2014:24:280-7.

7. Rosenkrantz AB, Verma S, Choyke P, Eberhardt SC, Eggener $\mathrm{SE}$, Gaitonde $\mathrm{K}$, et al. Prostate Magnetic Resonance Imaging and Magnetic Resonance Imaging Targeted Biopsy in Patients with a Prior Negative Biopsy: A Consensus Statement by AUA and SAR. J Urol. 2016;196:1613-8.

8. Schoots IG, Petrides N, Giganti F, Bokhorst LP, Rannikko A, Klotz $L$, et al. Magnetic resonance imaging in active surveillance of prostate cancer: a systematic review. Eur Urol. 2015;67:627-36.

9. Likert R. A technique for the measurement of attitudes. Archives of Psychology 1932;140:44-53.

10. Renard-Penna R, Mozer P, Cornud F, Barry-Delongchamps $\mathrm{N}$, Bruguière $\mathrm{E}$, Portalez $\mathrm{D}$, et al. Prostate Imaging Reporting and Data System and Likert Scoring System: Multiparametric MR Imaging Validation Study to Screen Patients for Initial Biopsy. Radiology. 2015;275:458-68.

11. Rosenkrantz AB, Kim S, Lim RP, Hindman N, Deng FM, Babb JS, et al. Prostate cancer localization using multiparametric MR imaging: comparison of Prostate Imaging Reporting and Data System (PI-RADS) and Likert scales. Radiology. 2013;269:482-92. 
12. Dickinson L, Ahmed HU, Allen C, Barentsz JO, Carey B, Futterer JJ, et al. Scoring systems used for the interpretation and reporting of multiparametric MRI for prostate cancer detection, Iocalization, and characterization: could standardization lead to improved utilization of imaging within the diagnostic pathway? J Magn Reson Imaging 2013;37:48-58.

13. American College of Radiology. MR Prostate Imaging Reporting an Data System version 2.0. <https://www.acr. org/Quality-Safety/Resources/PIRADS/> accessed January 05, 2017.

14. Rosenkrantz AB, Ginocchio LA, Cornfeld D, Froemming AT, Gupta RT, Turkbey B, et al. Interobserver Reproducibility of the PI-RADS Version 2 Lexicon: A Multicenter Study of Six Experienced Prostate Radiologists. Radiology. 2016;280:793-804

15. Muller BG, Shih JH, Sankineni S, Marko J, Rais-Bahrami S, George AK, et al. Prostate Cancer: Interobserver Agreement and Accuracy with the Revised Prostate Imaging Reporting and Data System at Multiparametric MR Imaging. Radiology. 2015;277:741-50.

16. Bains LJ, Studer UE, Froehlich JM, Giannarini G, Triantafyllou M, Fleischmann A, et al. Diffusion-weighted magnetic resonance imaging detects significant prostate cancer with a high probability: results of a prospective study with final pathology of prostates with and without cancer as the reference standard. J Urol 2014;192:1-21. Available at. <https://www.urotoday.com/recent-abstracts/urologiconcology/prostate-cancer/70901-diffusion-weightedmagnetic-resonance-imaging-detects-significant-prostatecancer-with-a-high-probability-results-of-a-prospectivestudy-with-final-pathology-of-prostates-with-and-withoutcancer-as-the-reference-standard-abstract.amp.html>

17. Nagel KN, Schouten MG, Hambrock T, Litjens GJ, Hoeks CM, ten Haken B, et al. Differentiation of prostatitis and prostate cancer by using diffusion-weighted MR imaging and MRguided biopsy at 3T. Radiology. 2013;267:164-72.
18. Woodfield CA, Tung GA, Grand DJ, Pezzullo JA, Machan JT, Renzulli JF 2nd. Diffusion-weighted MRI of peripheral zone prostate cancer: comparison of tumor apparent diffusion coefficient with Gleason score and percentage of tumor on core biopsy. AJR Am J Roentgenol. 2010;194:W316-22.

19. Anwar SS, Anwar Khan Z, Shoaib Hamid R, Haroon F, Sayani $\mathrm{R}$, Beg M, et al. Assessment of apparent diffusion coefficient values as predictor of aggressiveness in peripheral zone prostate cancer: comparison with Gleason score. ISRN Radiol. 2014;2014:263417.

20. Lebovici A, Sfrangeu SA, Feier D, Caraiani C, Lucan C, Suciu $\mathrm{M}$, et al. Evaluation of the normal-to-diseased apparent diffusion coefficient ratio as an indicator of prostate cancer aggressiveness. BMC Med Imaging. 2014;14:15.

21. Zhu J, Zhang J, Gao JY, Li JN, Yang DW, Chen M, et al. Apparent diffusion coefficient normalization of normal liver: Will it improve the reproducibility of diffusion-weighted imaging at different MR scanners as a new biomarker? Medicine (Baltimore). 2017;96:e5910.

22. Sadinski M, Medved M, Karademir I, Wang S, Peng Y, Jiang $\mathrm{Y}$, et al. Short-term reproducibility of apparent diffusion coefficient estimated from diffusion-weighted MRI of the prostate. Abdom Imaging. 2015;40:2523-8.

23. Rosenkrantz AB, Babb JS, Taneja SS, Ream JM. Proposed Adjustments to PI-RADS Version 2 Decision Rules: Impact on Prostate Cancer Detection. Radiology. 2017;283:119-29.

Correspondence address: Thais Caldara Mussi, MD Hospital Israelita Albert Einstein Albert Einstein, 627 Jardim Leonor, São Paulo, SP, 05652-900, Brasil Fax: + 5511 2151-4271 E-mail: thaiscaldara@gmail.com 